\title{
"LAS PRECIPITACIONES Y LOS PERÍODOS HÚMEDOS EN LA PROVINCIA DEL CHACO ENTRE LOS AÑOS 1961 Y 2005."
}

\author{
"RAINFALL AND WET PERIODS IN THE PROVINCE OF CHACO BETWEEN THE YEARS \\ 1961 AND 2005."
}

\author{
Lic.Pyszczek, Oscar Luis. UNNE-CONICET. \\ Prof. Pérez María Emilia. UNNE. Profesora Titular Cátedra Seminario de Fisiografía \\ Profesor Adjunta Cátedra Climatología
}

\begin{abstract}
RESUMEN
Las precipitaciones constituyen el elemento del clima que presenta la mayor variabilidad espaciotemporal en sus registros y, a su vez, constituye un condicionante de gran importancia en el desarrollo de las actividades productivas. El presente trabajo pretende indagar acerca del comportamiento de las precipitaciones en la provincia del Chaco, determinando los períodos húmedos y las posibles variaciones significativas que se pudieron registrar en los mismos, durante el período 1961/2005. Para ello se analizaron datos pluviométricos mensuales provenientes de cinco estaciones meteorológicas, seleccionadas en función de la extensión de los registros y su localización en el territorio Provincial.
\end{abstract}

Palabras claves: Precipitaciones; Períodos Húmedos; Montos Pluviométricos Mensuales.

\section{ABSTRACT}

Rainfall is the climatic element that has the highest spatial-temporal variability in their records and in turn is a major determinant in the development of productive activities. This paper aims to investigate about the behaviour of precipitation in the province of Chaco, by determining the wet and the significant variations that could be recorded in the same over the period 1961/2005. This will be analysed monthly rainfall data from five weather stations selected according to the length of the records and their location in the province.

Keywords: Precipitation; wet periods; Monthly rainfall amounts.

\section{INTRODUCCIÓN}

Las precipitaciones constituyen el elemento del clima que presenta mayor variabilidad espaciotemporaly, al mismo tiempo,debido a que sus fluctuaciones pueden provocar graves consecuencias en los circuitos productivos locales, condicionan el desarrollo de las actividades humanas en tanto sus montos sean deficitarios o excesivos. Por ello resulta trascendente el conocimiento de los montos y regímenes pluviométricos de la provincia del Chaco y, particularmente de los períodos húmedos, pues su tratamiento se encuentra retrasado en comparación con su opuesto representado por las sequías o períodos secos.

El reparto temporal y la distribución estacional de la lluvia permiten reconocer tendencias a través del análisis de los montos mensuales así como también determinar los períodos húmedos. El análisis de las lluvias se haya subordinado, principalmente, a la existencia de largas series de observación: en nuestro caso la serie comprende un intervalo de 45 años, correspondientes a la segunda mitad del

Publicado en formato digital: Lic. Oscar Luis Pyszczek y Prof. María Emilia Pérez.. LAS PRECIPITACIONES Y LOS PERÍODOS HÚMEDOS EN LA PROVINCIA DEL CHACO ENTRE LOS AÑOS 1961 Y 2005. Revista Geográfica Digital. IGUNNE. Facultad de Humanidades. UNNE. Año 10. № 19. Enero - Junio 2013. ISSN 16685180 Resistencia, Chaco. En: http://hum.unne.edu.ar/revistas/geoweb/default.htm 
siglo XX y primera década del siglo XXI en el Chaco. La longitud de la serie permitirá detectar la existencia de posibles variaciones en la distribución y comportamiento de las lluvias.

Los datos pluviométricos fueron suministrados por la Administración Provincial del Agua (A.P.A.), consistentes en los montos pluviométricos mensuales para la serie de años comprendidos entre 1961-2005, de los cuales se seleccionaron las series de datos para cinco localidades, distribuidas en el territorio Provincial. Las mismas son: Resistencia, Machagai, San Bernardo, Juan José Castelli y Los Frentones.

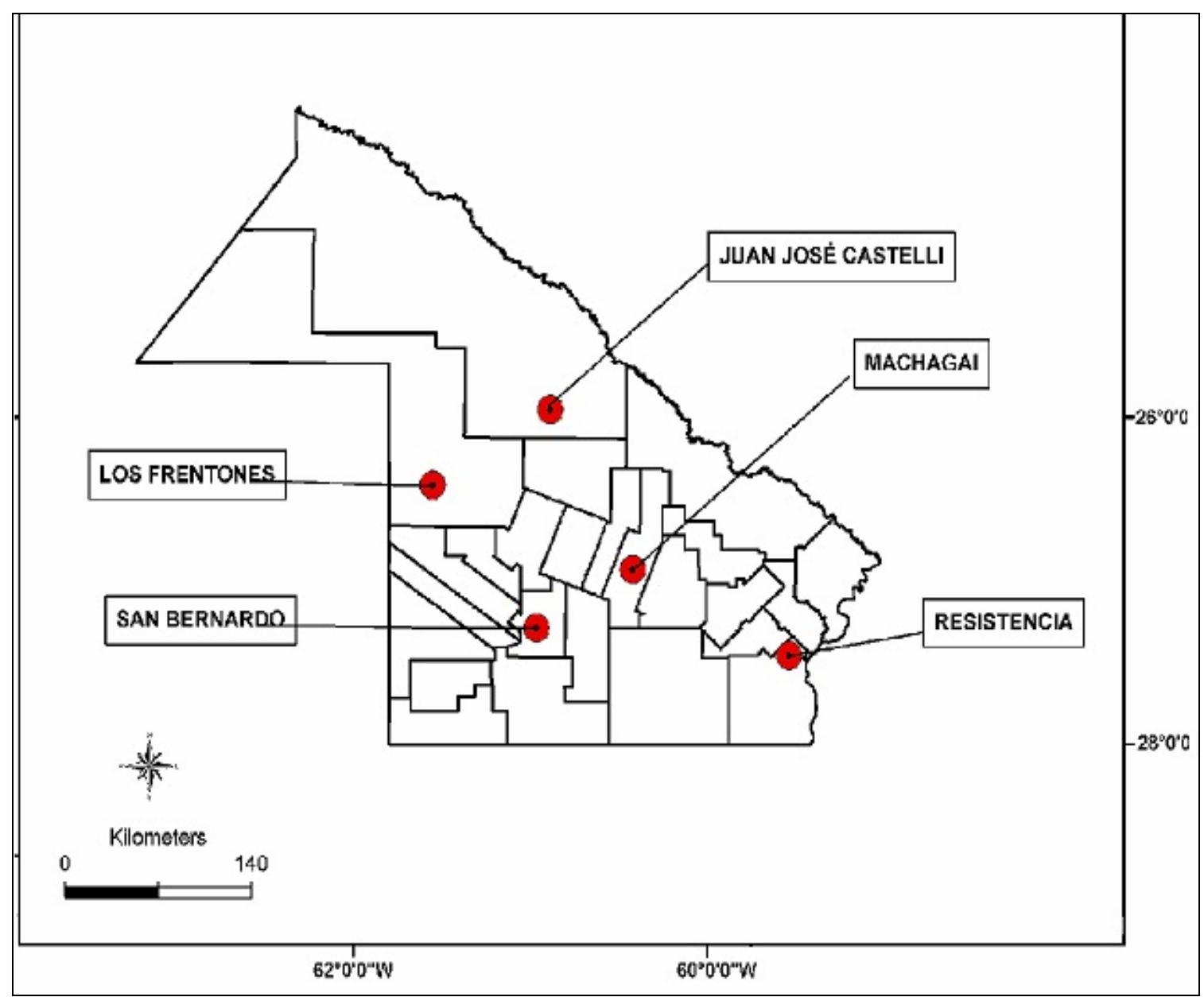

Fig.1. Localización de los puestos pluviométricos.

\section{MATERIALES Y MÉTODOS}

Las estaciones pluviométricas fueron seleccionadas en función de la extensión temporal de sus registros pluviométricos y de la localización en el territorio provincial, evitando escoger puestos próximos entre sí y abarcar la mayor extensión provincial posible. Una vez seleccionadas y localizadas, se procedió al tratamiento y normalización de los datos estadísticos.

La información suministrada por la Administración Provincial del Agua (A.P.A.) se encuentra tabulada según año hidrológico ${ }^{1}$, motivo por el cual fue necesario ordenar los datos por año calendario (desde

${ }^{1}$ El año hidrológico en nuestra provincia se extiende desde el mes de Septiembre al mes de Agosto del año siguiente.

Publicado en formato digital: Lic. Oscar Luis Pyszczek y Prof. María Emilia Pérez.. LAS PRECIPITACIONES Y LOS PERÍODOS HÚMEDOS EN LA PROVINCIA DEL CHACO ENTRE LOS AÑOS 1961 Y 2005. Revista Geográfica Digital. IGUNNE. Facultad de Humanidades. UNNE. Año 10. № 19. Enero - Junio 2013. ISSN 16685180 Resistencia, Chaco. En: http://hum.unne.edu.ar/revistas/geoweb/default.htm 
Revista Geográfica Digital. IGUNNE. Facultad de Humanidades. UNNE. Año 10. № 19. Enero - Junio 2013.

ISSN 1668-5180 Resistencia, Chaco

los meses de enero a diciembre); y calcular los montos pluviométricos anuales que se presentan en el siguiente cuadro.

$\underline{\text { Tabla No } 1 . ~ M o n t o s ~ p l u v i o m e ́ t r i c o s ~ m e d i o s ~ m e n s u a l e s ~ y ~ a n u a l e s ~(e n ~ m m) . ~ P e r i ́ o d o ~ 1961 / 2005 ~}$

\begin{tabular}{|l|c|c|c|c|c|c|c|c|c|c|c|c|c|}
\hline \multicolumn{1}{|c|}{ ESTACION } & E & F & M & A & M & J & J & A & S & O & N & D & ANUAL \\
\hline Resistencia & 165 & 158 & 167 & 177 & 84 & 57 & 40 & 44 & 64 & 132 & 150 & 147 & $\mathbf{1 3 8 5}$ \\
\hline Machagai & 138 & 138 & 152 & 143 & 58 & 32 & 22 & 27 & 44 & 102 & 138 & 127 & $\mathbf{1 1 2 1}$ \\
\hline San Bernardo & 131 & 135 & 164 & 140 & 49 & 19 & 22 & 17 & 48 & 82 & 114 & 115 & $\mathbf{1 0 3 6}$ \\
\hline J.J.Castelli & 122 & 102 & 116 & 107 & 44 & 19 & 16 & 16 & 34 & 75 & 107 & 107 & $\mathbf{8 6 5}$ \\
\hline Los Frentones & 128 & 125 & 122 & 94 & 36 & 12 & 15 & 11 & 31 & 64 & 101 & 124 & $\mathbf{8 6 3}$ \\
\hline
\end{tabular}

FUENTE: Tabla elaborada a partir de los datos suministrados por la A.P.A.

Según la Administración Provincial del Agua, cuando las precipitaciones de un mes son inferiores al $50 \%$ del promedio mensual de la serie de años, es considerado un mes seco.Cuando esta tendencia se mantiene como mínimo por tres meses consecutivos se considera período de Sequía.

Es aquí, donde se presenta el primer obstáculo en la búsqueda de los períodos de excesos hídricos: la mayoría de las investigaciones establecen criterios para definir los meses secos e intervalos secos mientras que no han sido trabajado suficientemente los criterios para establecer meses húmedos o períodos húmedos.

Para superar dicho obstáculo se ha recurrido al método estadístico de los Quintiles. El método por Quintiles "permite distinguir cuatro quintiles que dividen la serie en cinco subconjuntos de igual tamaño" (Fernández García, 1995), que comprenden:

- Q1: el 20\% de las observaciones menores al primer quintil y el $80 \%$ superior a éste.

- Q4: el 20\% de las observaciones mayores al cuarto quintil y el $80 \%$ inferior a éste.

- Q2: el $40 \%$ de las observaciones menores al segundo quintil y el $60 \%$ de las observaciones superior a éste.

- Q3: el $40 \%$ de las observaciones superiores al tercer quintil y el $60 \%$ de las observaciones inferiores a éste.

Las Quintilas son utilizadas por Institutos y Servicios Nacionales de Meteorología (como por ejemplo el de España y el de Argentina), como criterio de clasificación de los años en muy secos, secos, normales, lluviosos y muy lluviosos, en función de los montos pluviométricos anuales. Para llevar a cabo la división de los datos a través del método de Quintiles es necesario en primer lugar ordenar los datos en forma creciente o decreciente según las necesidades.

La definición de los quintiles se realizó a través de la fórmula:

$$
i=\underline{(2 n \cdot c)+1}
$$

2

Donde:

$\mathrm{i}$ = Rango del elemento o lugar que ocupa según su valor;

$\mathrm{n}=$ total de valores de la muestra;

$\mathrm{c}=$ Frecuencia Relativa de la cuantila.

Publicado en formato digital: Lic. Oscar Luis Pyszczek y Prof. María Emilia Pérez.. LAS PRECIPITACIONES Y LOS PERÍODOS HÚMEDOS EN LA PROVINCIA DEL CHACO ENTRE LOS AÑOS 1961 Y 2005. Revista Geográfica Digital. IGUNNE. Facultad de Humanidades. UNNE. Año 10. No 19. Enero - Junio 2013. ISSN 16685180 Resistencia, Chaco. En: http://hum.unne.edu.ar/revistas/geoweb/default.htm 


\section{OBJETIVOS}

-Analizar la variabilidad en el espacio y en el tiempo de las precipitaciones en el territorio de la Provincia del Chaco en el período 1961-2005.

-Identificar los años lluviosos y muy lluviosos, mediante el método de quintiles.

-Determinar los períodos húmedos existentes (su duración, magnitud y frecuencia).

\section{RESULTADOS}

La aplicación del método de Quintiles, considerando los montos pluviométricos anuales de la serie estadística, establececuatro montos pluviométricos que dividen a las serie en $\mathbf{5}$ (Cinco) grupos. Los montos pluviométricos anuales han sido ordenados previamente de modo creciente, de manera tal que la disposición de los mismos se asocie a los conjuntos de años Muy Secos; Secos; Normales; Lluviosos y Muy Lluviosos. (Ver tabla $n^{0} 5$ ).

Los umbrales máximos y mínimos de montos pluviométricos, para el grupo de años Muy lluviosos, se encuentran expresados en la Tabla $N^{\circ} 2$. Si observamos dichos umbrales podemos apreciar una notable disminución de los mismos a medida que avanzamos en dirección Oeste en la provincia del Chaco (en sentido descendente de la columna). Es decir que no solamente disminuyen los montos de precipitaciones sino que también las diferencias entre los umbrales.

Tabla № 2.Umbrales máximos y mínimos de Precipitaciones en grupo de años muy Lluviosos, Período 1961-2005.

\begin{tabular}{|l|c|c|c|}
\hline Estación Pluviométrica & Umbral Máximo & Umbral Mínimo & $\begin{array}{c}\text { Diferencias entre } \\
\text { Umbrales Máx. } \boldsymbol{y} \\
\text { Mín. }\end{array}$ \\
\hline Resistencia & $2218 \mathrm{~mm}$ & $1626 \mathrm{~mm}$ & $592 \mathrm{~mm}$ \\
\hline Machagai & $1908 \mathrm{~mm}$ & $1451 \mathrm{~mm}$ & $457 \mathrm{~mm}$ \\
\hline San Bernardo & $1715 \mathrm{~mm}$ & $1245 \mathrm{~mm}$ & $470 \mathrm{~mm}$ \\
\hline Los Frentones & $1427 \mathrm{~mm}$ & $989 \mathrm{~mm}$ & $438 \mathrm{~mm}$ \\
\hline Juan José Castelli & $1301 \mathrm{~mm}$ & $1005 \mathrm{~mm}$ & $296 \mathrm{~mm}$ \\
\hline
\end{tabular}

FUENTE: Tabla elaborada a partir de los datos suministrados por la A.P.A.

A fines de nuestros objetivos, sólose han tenido en cuenta los quintiles 3 y 4 (Q3 y Q4) que son los que determinan los umbrales de los grupos de años lluviosos y muylluviosos. Los valores hallados para la serie de estudio son:

Tabla № 3. Quintiles para cada Estación Meteorológica Seleccionada.

\begin{tabular}{|c|c|c|c|c|c|c|c|c|c|}
\hline \multicolumn{2}{|c|}{ RESISTENCIA } & \multicolumn{2}{c|}{ MACHAGAI } & \multicolumn{2}{c|}{$\begin{array}{c}\text { SAN } \\
\text { BERNARDO }\end{array}$} & \multicolumn{2}{c|}{ J.J. CASTELLI } & \multicolumn{2}{c|}{ LOS } \\
FRENTONES \\
\hline Q3 & Q4 & Q3 & Q4 & Q3 & Q4 & Q3 & Q4 & Q3 & Q4 \\
\hline 1421 & 1612,5 & 1121,5 & 1430 & 1056,5 & 1234 & 848 & 1000,5 & 888,5 & 987 \\
\hline
\end{tabular}

FUENTE: Tabla elaborada a partir de los datos suministrados por la A.P.A.

La importancia de los grupos obtenidos se evidencia en la distribución de los años (tanto Lluviosos como Muy Lluviosos) en la serie total, lo que a su vez permite advertir, una serie de años consecutivos que constituyen los períodos Húmedosde mayor pluviosidad en distintos sectores de la Provincia del Chaco. 
Revista Geográfica Digital. IGUNNE. Facultad de Humanidades. UNNE. Año 10. № 19. Enero - Junio 2013. ISSN 1668-5180 Resistencia, Chaco

Tabla № 4. Años Lluviosos y Muy lluviosos, por Estación Meteorológica (en mm). Período 1965-2005.

\begin{tabular}{|c|c|c|c|c|c|c|c|c|c|}
\hline \multicolumn{2}{|c|}{ RESISTENCIA } & \multicolumn{2}{c|}{ MACHAGAI } & \multicolumn{2}{c|}{ SAN BERNARDO } & \multicolumn{2}{c|}{ J.J. CASTELLI } & \multicolumn{2}{c|}{ LOS } \\
\hline AÑO & MONTO & AÑO & MONTO & AÑO & MONTO & AÑO & MONTO & AÑO & MONTO \\
\hline 1961 & 1599 & $\mathbf{1 9 6 1}$ & 1202 & $\mathbf{1 9 6 1}$ & 1131 & $\mathbf{1 9 6 2}$ & 948 & $\mathbf{1 9 6 1}$ & 989 \\
\hline 1965 & 1626 & $\mathbf{1 9 6 5}$ & 1451 & & & $\mathbf{1 9 6 5}$ & 996 & $\mathbf{1 9 6 4}$ & 943 \\
\hline & & $\mathbf{1 9 6 6}$ & 1462 & $\mathbf{1 9 7 2}$ & 1214 & $\mathbf{1 9 6 7}$ & 1038 & $\mathbf{1 9 6 6}$ & 968 \\
\hline $\mathbf{1 9 7 2}$ & 1523 & $\mathbf{1 9 6 7}$ & 1298 & $\mathbf{1 9 7 3}$ & 1153 & $\mathbf{1 9 6 8}$ & 1005 & $\mathbf{1 9 6 8}$ & 906 \\
\hline $\mathbf{1 9 7 3}$ & 2015 & & & $\mathbf{1 9 7 7}$ & 1130 & $\mathbf{1 9 6 9}$ & 906 & & \\
\hline $\mathbf{1 9 7 5}$ & 1519 & $\mathbf{1 9 7 3}$ & 1467 & & & & & $\mathbf{1 9 7 1}$ & 894 \\
\hline & & & & $\mathbf{1 9 8 1}$ & 1270 & $\mathbf{1 9 7 5}$ & 964 & $\mathbf{1 9 7 3}$ & 985 \\
\hline $\mathbf{1 9 8 2}$ & 1562 & $\mathbf{1 9 8 1}$ & 1229 & $\mathbf{1 9 8 2}$ & 1057 & $\mathbf{1 9 7 8}$ & 857 & $\mathbf{1 9 7 4}$ & 890 \\
\hline $\mathbf{1 9 8 4}$ & 1562 & $\mathbf{1 9 8 4}$ & 1190 & $\mathbf{1 9 8 3}$ & 1069 & & & $\mathbf{1 9 7 7}$ & 955 \\
\hline $\mathbf{1 9 8 5}$ & 1477 & $\mathbf{1 9 8 6}$ & 1480 & $\mathbf{1 9 8 4}$ & 1715 & $\mathbf{1 9 8 0}$ & 1242 & $\mathbf{1 9 7 9}$ & 1016 \\
\hline $\mathbf{1 9 8 6}$ & 2218 & $\mathbf{1 9 8 9}$ & 1126 & $\mathbf{1 9 8 5}$ & 1403 & $\mathbf{1 9 8 1}$ & 1106 & & \\
\hline $\mathbf{1 9 8 7}$ & 1429 & & & $\mathbf{1 9 8 6}$ & 1581 & $\mathbf{1 9 8 5}$ & 1132 & $\mathbf{1 9 8 0}$ & 1048 \\
\hline $\mathbf{1 9 8 9}$ & 1513 & $\mathbf{1 9 9 0}$ & 1292 & $\mathbf{1 9 8 9}$ & 1257 & $\mathbf{1 9 8 6}$ & 1056 & $\mathbf{1 9 8 3}$ & 978 \\
\hline & & $\mathbf{1 9 9 1}$ & 1409 & & & & & $\mathbf{1 9 8 6}$ & 1427 \\
\hline $\mathbf{1 9 9 0}$ & 1933 & $\mathbf{1 9 9 2}$ & 1603 & $\mathbf{1 9 9 1}$ & 1223 & $\mathbf{1 9 9 1}$ & 1044 & & \\
\hline $\mathbf{1 9 9 2}$ & 1663 & $\mathbf{1 9 9 4}$ & 1908 & $\mathbf{1 9 9 2}$ & 1141 & $\mathbf{1 9 9 2}$ & 920 & $\mathbf{1 9 9 2}$ & 1047 \\
\hline $\mathbf{1 9 9 4}$ & 1665 & $\mathbf{1 9 9 6}$ & 1582 & $\mathbf{1 9 9 6}$ & 1452 & $\mathbf{1 9 9 4}$ & 947 & $\mathbf{1 9 9 5}$ & 980 \\
\hline $\mathbf{1 9 9 6}$ & 2007 & $\mathbf{1 9 9 7}$ & 1241 & $\mathbf{1 9 9 8}$ & 1278 & $\mathbf{1 9 9 6}$ & 995 & $\mathbf{1 9 9 6}$ & 1022 \\
\hline $\mathbf{1 9 9 7}$ & 1471 & $\mathbf{1 9 9 8}$ & 1537 & & & & & $\mathbf{1 9 9 8}$ & 1015 \\
\hline $\mathbf{1 9 9 8}$ & 1741 & & & $\mathbf{2 0 0 0}$ & 1245 & $\mathbf{2 0 0 0}$ & 1108 & & \\
\hline & & $\mathbf{2 0 0 0}$ & 1327 & $\mathbf{2 0 0 1}$ & 1098 & $\mathbf{2 0 0 2}$ & 1301 & $\mathbf{2 0 0 0}$ & 1016 \\
\hline $\mathbf{2 0 0 2}$ & 2090 & $\mathbf{2 0 0 2}$ & 1733 & $\mathbf{2 0 0 2}$ & 1591 & & & $\mathbf{2 0 0 2}$ & 1216 \\
\hline & & & & & & $\mathbf{2 0 0 3}$ & 920 & & \\
\hline
\end{tabular}

FUENTE: Ídem tabla 1.

La distribución de los años lluviosos y muy lluviosos, determinados para las localidades seleccionadas, permite advertir las siguientes características en el comportamiento de los montos anuales de precipitación:

\section{RESISTENCIA}

La distribución de los años lluviosos y muy lluviosos presenta una disposición temporal caracterizada, en las primeras décadas de la serie, por montos pluviométricos no muy elevados, y que denotan algunos años Lluviosos aislados, con recurrencia espaciada en el tiempo $(1961,1965,1972,1973$ y 1975). Sus montos se encuentran entre los 1500 y $2000 \mathrm{~mm}$ anuales.

Desde 1982 a 1998, la ocurrencia más frecuente de años lluviosos y muy lluviosos nos permite inferir en una mayor actividad de los centros de acción atmosféricos y el mayor aporte de masas de aire con alto contenido de humedad, originando precipitaciones importantes, que en algunos casos superaron los $2000 \mathrm{~mm}$. anuales. En la década de 1980, la distribución es bastante más continua que en la década de 1990, que resulta un poco más dispersa, si bien se encuentran separados o intercalados por años normales o secos. La distribución y frecuencia de estos altos montos durante estos años, nos estaría indicando el inicio de un ciclo húmedo, el que pareciera haber finalizado a partir de los años 2000 .

Publicado en formato digital: Lic. Oscar Luis Pyszczek y Prof. María Emilia Pérez.. LAS PRECIPITACIONES Y LOS PERÍODOS HÚMEDOS EN LA PROVINCIA DEL CHACO ENTRE LOS AÑOS 1961 Y 2005. Revista Geográfica Digital. IGUNNE. Facultad de Humanidades. UNNE. Año 10. № 19. Enero - Junio 2013. ISSN 16685180 Resistencia, Chaco. En: http://hum.unne.edu.ar/revistas/geoweb/default.htm 


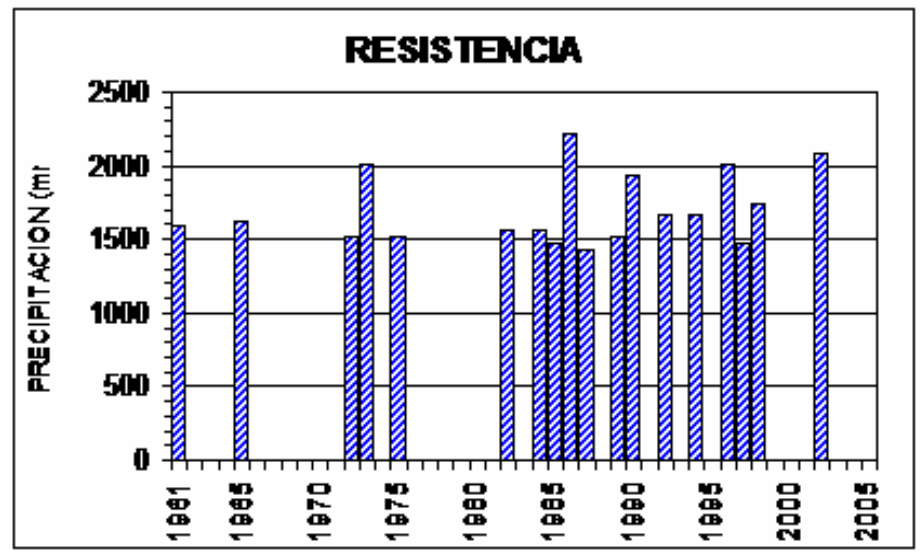

Gráfico 1. Distribución de años lluviosos y muy lluviosos para la estación Resistencia.

\section{MACHAGAI}

Si bien la distribución temporal de los años Lluviosos y Muy Lluviosos, en esta localidad, es similar a lo obtenido en la estación de Resistencia; presenta matices que lo diferencian. Los datos arrojan que existen intervalos de tiempo diferentes en cuanto a la ocurrencia de precipitaciones:el primero estaría conformado por las dos primeras décadas de la serie (1961 a 1980) en forma consecutiva, caracterizado por la menor ocurrencia de años Lluviosos, cuyos montos no sobrepasan los $1500 \mathrm{~mm}$., a diferencia de Resistencia cuyos montos son superiores.El segundo grupo, más extenso que el anterior,lo componen los años 1980 hasta el 2002,donde el rasgo característico, es la aparición de mayor cantidad de años Lluviosos y Muy Lluviosos intercalados con otros normales y/o secos.

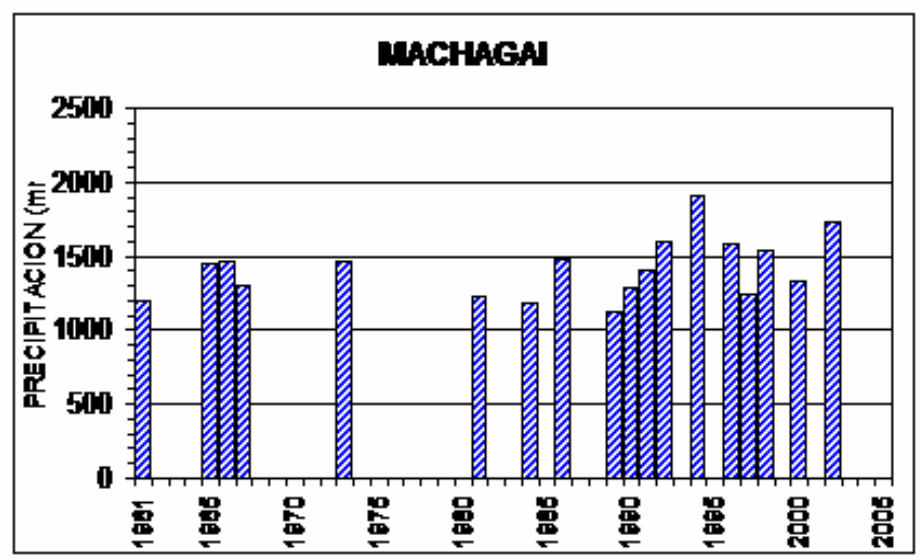

Gráfico 2. Distribución de años lluviosos y muy lluviosos para la estación Machagai.

La disposición de los mismos también difiere de lo descrito para el caso de Resistencia, evidenciándose una mayor regularidad de ocurrencia para los años de la década de 1990.

\section{SAN BERNARDO}

El ritmo en cuanto a la ocurrencia de los años Lluviosos y Muy Lluviosos, presenta patrones de distribución similares a los casos anteriores, pero con diferencias en cuanto a los montos obtenidos, debido a la disminución de los mismos en sentido oeste

Nuevamente se observa que desde 1961 a 1980 sólo se registran tres años lluviosos en consonancia con las anteriores estaciones, pero con montos menores. Entre 1980- 2000 es posible observar un 
ciclo de ocurrencias de montos pluviométricos correspondientes a los años muy lluviosos en el cual se destaca el año 1984.

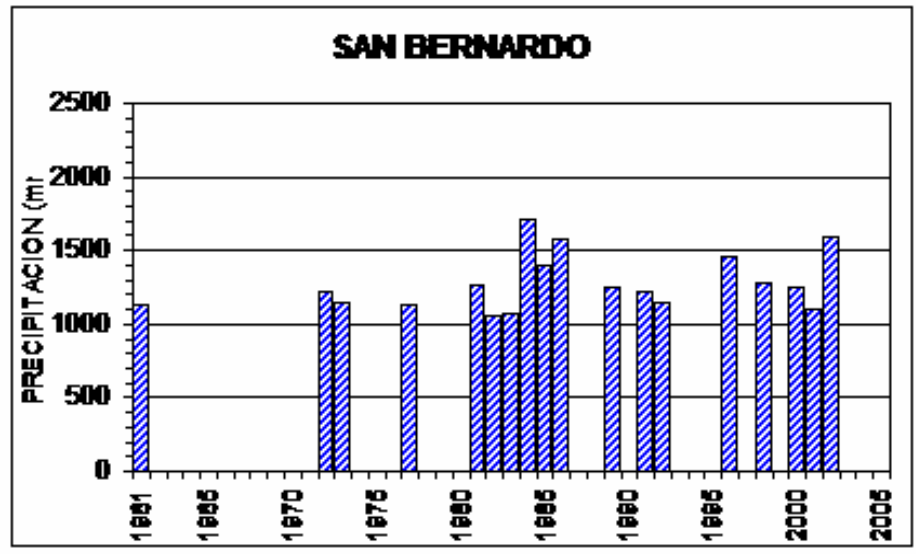

Gráfico 3. Distribución de años lluviosos y muy lluviosos para la estación San Bernardo.

Luego de 1986, los años lluviosos se presentan de modo intercalado, más espaciados, hasta 1995 donde comienza nuevamente un lapso más regular de ocurrencia con un mayor agrupamiento.

Un aspecto a tener cuenta, radica que el período de mayor agrupamiento de los años Lluviosos se registra entre 1981-1986, de forma coincidente con el obtenido en Resistencia.

\section{JUAN JOSE CASTELLI}

Muestra una distribución mucho más segmentada y a su vez, más regular que las anteriores. A diferencia de los datos analizados hasta el momento, la distribución de los años Lluvioso y Muy Lluviosos muestra una frecuencia esporádica, lo cual no permite diferenciar agrupamientos importantes de años con alta pluviosidad.

En los años 1960 se evidencia una serie de años lluviosos intercalados con normales y secos, hecho que diferencia a esta década de la que le sigue, en la cual disminuye la frecuencia de años de alta pluviosidad.

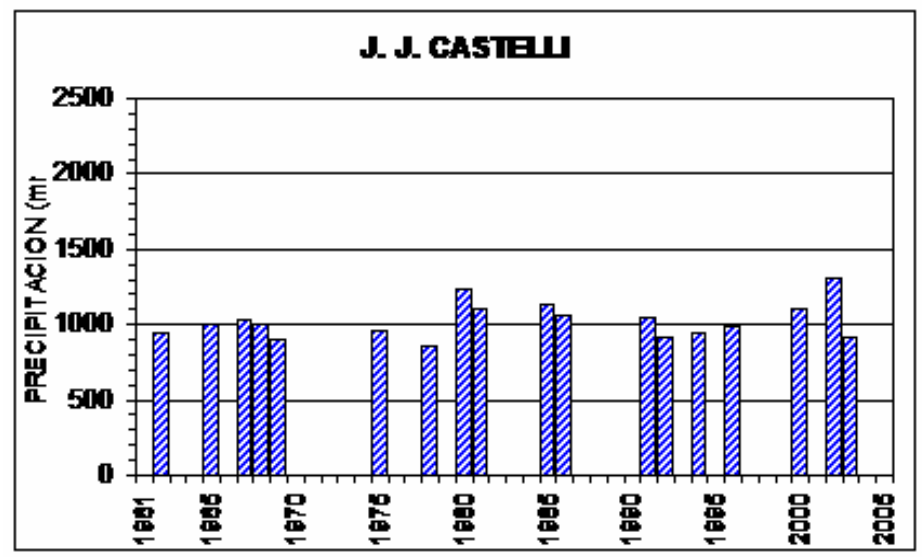

Gráfico 4. Distribución de años lluviosos y muy lluviosos para la estación J.J.Castelli.

Desde los años 1980-2005, la ocurrencia continúa siendo segmentada y regular con montos que oscilan entre los 1000 y $1300 \mathrm{~mm}$ anuales. 


\section{LOS FRENTONES}

En lo que respecta a la precipitación de dicha localidad, la distribución de los montos denota un comportamiento similar a lo descrito para Juan José Castelli, y al igual que esta última presenta una distribución regular a lo largo de la serie de estudio.

Desde 1961 a 1986 predominan los años de tipo lluvioso con notable regularidad en cuanto a la distribución, separados por uno o dos años normales o secos. En 1986 se registra el mayor monto pluviométrico de la serie dentro de los años Lluviosos con montos que se acercan a los $1500 \mathrm{~mm}$, considerablemente mayor si tenemos en cuenta que esta localidad posee un valor anual promedio de $825 \mathrm{~mm}$.

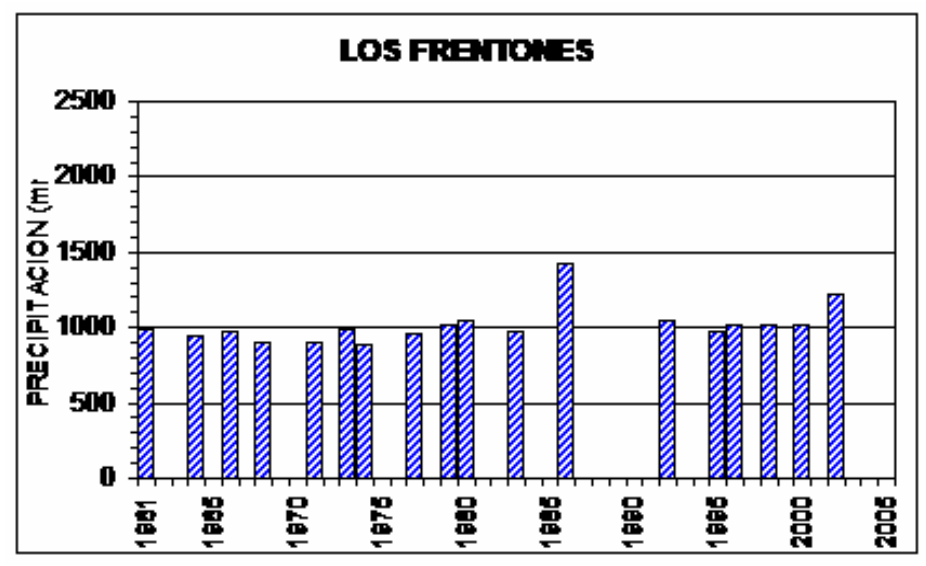

Gráfico 5. Distribución de años lluviosos y muy lluviosos para la estación Los Frentones.

La serie continúa la tendencia de regularidad de ocurrencia hasta finalizar la misma. El único lapso donde se observa una discontinuidad marcada corresponde a los años 1987-1991.

\section{CONCLUSIONES}

Más allá de las diferencias entre las localidades, concernientes a los años con excesos de precipitaciones, hay que destacar que en los cinco casos de estudio, se advierte un comportamiento similar en los registros pluviométricos al momento que se comparan sus tendenciaspolinómicas de 4 términos. Esto afirma los rasgos subyacentes del clima Templado Cálido o Subtropical predominante en la Provincia del Chaco.

Se evidencia que las décadas de 1960 y 1970 fueron menos lluviosas que las de 1980 y 1990, y que a partir del año 2003, en todos los casos, los montos anuales presentan una marcada disminución. Se advierte al mismo tiempo, que los registros de la localidad de Castelli, si bien presentan el mismo ritmo que las otras localidades, los montos son más uniformes a lo largo del período de estudio, situación que también se corresponde con los umbrales máximos y mínimos expuestos en la Tabla $\mathrm{N}^{0} 2$.

Las causas de esta distribución espacial y temporal, están vinculadas al hecho que toda la provincia del Chaco está sujeta a las mismas condiciones genéticas que determinan las lluvias.Las diferencias detectadas podrían deberse a dos causas principales: por un lado, a las lluvias de verano, las cuales son predominante de origen convectivo (inestabilidad local) y generan lluvias o chaparrones aislados intensos y muy localizados. En segundo lugar, debemos tener en cuenta que los datos aportados por la A.P.A.(administración Provincial del Agua) son registros obtenidos en puestos pluviométricos provinciales, la mayoría de los que se recogen en estaciones policiales y por personal que a veces no tiene el entrenamiento necesario, y por lo tanto, los mismos podrían adolecer de errores de lectura y medición.

Publicado en formato digital: Lic. Oscar Luis Pyszczek y Prof. María Emilia Pérez.. LAS PRECIPITACIONES Y LOS PERÍODOS HÚMEDOS EN LA PROVINCIA DEL CHACO ENTRE LOS AÑOS 1961 Y 2005. Revista Geográfica Digital. IGUNNE. Facultad de Humanidades. UNNE. Año 10. No 19. Enero - Junio 2013. ISSN 16685180 Resistencia, Chaco. En: http://hum.unne.edu.ar/revistas/geoweb/default.htm 
La variabilidad que presentan las precipitaciones, hace difícil la detección de una tendencia firme en los datos. No obstante las oscilaciones espacio-temporales, posibilitaron por ejemplo, la expansión de la frontera agrícola en el oeste de la provincia, multiplicándose, los campos en donde se cultiva la soja.

Las deficiencias pluviométricas sin duda, ejercen una acción muy negativa en las actividades productivas primarias fundamentalmente, pero así como también se suceden períodos importantes de lluvias, se genera una especie de juego de equilibrio, regulación o compensación del sistema. Esta compensación del sistema climático es visible en los datos estadísticos de las series antes tratadas. Es por ello que no necesariamente ni las fuertes sequías vivida en los últimos años en la provincia ni los elevados montos de los años '80y '90, se vinculen al proceso de Cambio Climático, tan mencionado en los últimos tiempos y tal vez responda al juego de compensación hídrica.

"El cómo enfrentar estos fenómenos se relaciona con las condiciones sociales, económicas, culturales, geofísicas y meteorológicas en las que éstos se desarrollan. Por ello, la comprensión y las medidas de mitigación de los expertos y agricultores para enfrentar las sequías, difieren notablemente." (Informe de la ONU sobre Cambio Climático. 2009).

El desafío será afrontar las adversidades que presentan los ciclos de precipitaciones, en este caso con la mayor creatividad posible y un esfuerzo compartido entre los sectores involucrados.

\section{BIBLIOGRAFIA:}

- BARRY R. G. Y CHORLEY R. J. “Atmósfera, Tiempo y Clima”. Omega, Barcelona, 1972.

- BIROT P. “Tratado de Geografía Física General”. Vicens-Vives, Barcelona, 1962.

- BOLOGNESI, E. (1971). "Análisis de criterios para clasificar totales mensuales de precipitación aplicados a series de la República Argentina". En: Meteorológica. Buenos Aires, Centro Argentino de Meteorólogos, Vol. II. Ns ${ }^{\circ}$ 1, 2, y 3.

- BRUNIARD ENRIQUe D. "El Clima de las Planicies del Norte Argentino". UNNE, 1981.

- BRUNIARD ENRIQUE D. "Geografía Pluviométrica de la Provincia del Chaco". En Rev. Nordeste No 4. Fac. de Humanidades Resistencia, Chaco, 1962.

- CUADRAT, J. M. y PITA, M. F. (1997). "Climatología”. Madrid, Cátedra.

- FERnÁNDEZ GARCíA, FELIPE. "Manual de Climatología Aplicada. Clima, Medio Ambiente y Planificación". Síntesis, Madrid, 1996.

- HOFFMANN, J. A. (1988). "Las Variaciones Climáticas Ocurridas en la Argentina desde Fines del Siglo Pasado hasta el Presente". En: El Deterioro del Ambiente en la Argentina. Buenos Aires, FECIC.

- MILLER, AUSTIN A. “Climatología”. Omega. Barcelona,1951.

- MINETTI, J. y VARGAS, W. (1997). "Trends and Jumps in the annual precipitation in South America, south of the $15^{\circ}$ Sur". En: Atmósfera. México, Vol. 11, № 4.

PÉREZ, MARÍA EMILIA. "Vulnerabilidad Física. La variabilidad de las precipitaciones y los riesgos de sequías e inundaciones en el Norte Argentino durante los años 1951 a1991". En: "Aportes conceptuales y empíricos de la vulnerabilidad global". Foschiatti, Ana María (Autor/Compilador). Corrientes, 2008/2009, Universidad Nacional del Nordeste. Cap. 2, pp. 42/83. ISBN 978-950-656-1222 [Versión digital]. 
Tabla n 4 Determinación de los Años Lluviosos y Muy Lluviosos seqún el Método de Quintiles.

\begin{tabular}{|c|c|c|c|c|c|c|c|c|c|}
\hline \multicolumn{2}{|c|}{ RESISTENCIA } & \multicolumn{2}{|c|}{ J.J. CASTELLI } & \multicolumn{2}{|c|}{ LOS FRENTONES } & \multicolumn{2}{|c|}{ SAN BERNARDO } & \multicolumn{2}{|c|}{ MACHAGAI } \\
\hline AÑO & TOTAL & AÑO & TOTAL & AÑO & TOTAL & AÑO & TOTAL & AÑO & TOTAL \\
\hline 1988 & 941 & 1976 & 475 & 1988 & 341 & 2004 & 473 & 1976 & 486 \\
\hline 1971 & 1021 & 2001 & 525 & 2004 & 395 & 1988 & 598 & 1971 & 615 \\
\hline 1974 & 1026 & 2005 & 607 & 1989 & 559 & 1997 & 658 & 1980 & 723 \\
\hline 1962 & 1031 & 1997 & 639 & 1976 & 577 & 1978 & 703 & 1970 & 727 \\
\hline 1968 & 1038 & 1995 & 648 & 2001 & 579 & 1962 & 716 & 2001 & 728 \\
\hline 2004 & 1050 & 2004 & 666 & 1997 & 597 & 1993 & 727 & 1999 & 806 \\
\hline 1979 & 1055 & 1963 & 681 & 1978 & 605 & 1968 & 733 & 1978 & 812 \\
\hline 1999 & 1101 & 1973 & 685 & 2005 & 606 & 2003 & 756 & 1977 & 814 \\
\hline 1993 & 1103 & 1990 & 690 & 1999 & 613 & 1999 & 761 & 2005 & 845 \\
\hline 1969 & 1108 & 1983 & 694 & 1972 & 624 & 1976 & 794 & 1969 & 850 \\
\hline 1967 & 1118 & 1970 & 699 & 1962 & 664 & 1963 & 837 & 1968 & 879 \\
\hline 1964 & 1119 & 1999 & 705 & 1993 & 681 & 1995 & 901 & 1964 & 886 \\
\hline 2003 & 1147 & 1993 & 711 & 2003 & 697 & 1971 & 909 & 1972 & 886 \\
\hline 1976 & 1162 & 1988 & 718 & 1963 & 713 & 1967 & 931 & 1975 & 899 \\
\hline 1963 & 1189 & 1984 & 730 & 1969 & 730 & 1974 & 944 & 1988 & 936 \\
\hline 1970 & 1204 & 1987 & 731 & 1990 & 742 & 1990 & 958 & 1962 & 937 \\
\hline 2005 & 1220 & 1974 & 737 & 1967 & 767 & 1987 & 967 & 1995 & 938 \\
\hline 1995 & 1230 & 1982 & 740 & 1970 & 769 & 1994 & 967 & 2003 & 967 \\
\hline 1978 & 1254 & 1964 & 747 & 1994 & 784 & 1979 & 977 & 1963 & 970 \\
\hline 1980 & 1271 & 1966 & 751 & 1991 & 803 & 1970 & 996 & 1993 & 971 \\
\hline 1981 & 1317 & 1971 & 789 & 1987 & 812 & 1969 & 1030 & 1987 & 1016 \\
\hline 2001 & 1332 & 1989 & 808 & 1981 & 831 & 1964 & 1038 & 1983 & 1041 \\
\hline 1977 & 1363 & 1998 & 808 & 1975 & 844 & 1980 & 1038 & 1985 & 1051 \\
\hline 2000 & 1367 & 1979 & 810 & 1982 & 853 & 1966 & 1038 & 2004 & 1069 \\
\hline 1983 & 1400 & 1961 & 830 & 1965 & 878 & 1965 & 1053 & 1982 & 1094 \\
\hline
\end{tabular}
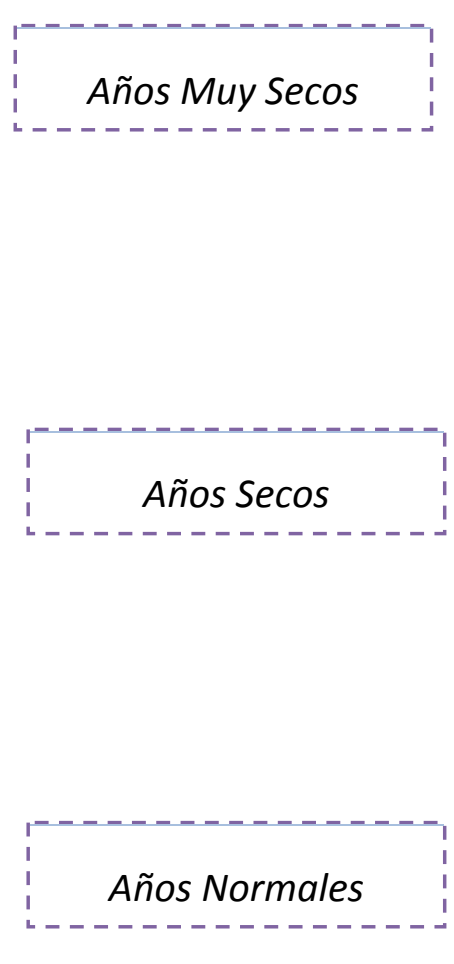

Publicado en formato digital: Lic. Oscar Luis Pyszczek y Prof. María Emilia Pérez.. LAS PRECIPITACIONES Y LOS PERÍODOS HÚMEDOS EN LA PROVINCIA DEL CHACO ENTRE LOS AÑOS 1961 Y 2005. Revista Geográfica Digital. IGUNNE. Facultad de Humanidades. UNNE. Año 10. № 19. Enero - Junio 2013. ISSN 1668-5180 Resistencia, Chaco. En: http://hum.unne.edu.ar/revistas/geoweb/default.htm 


\begin{tabular}{|l|l|l|l|l|l|l|l|l|l|}
\hline 1966 & 1413 & 1972 & 830 & 1984 & 881 & 1975 & 1056 & 1979 & 1102 \\
\hline 1991 & 1413 & 1977 & 839 & 1985 & 887 & -- & - & 1974 & 1117 \\
\hline 1987 & 1429 & 1978 & 857 & 1974 & 890 & 1982 & 1057 & 1989 & 1126 \\
\hline 1997 & 1471 & 1969 & 906 & 1971 & 894 & 1983 & 1069 & 1984 & 1190 \\
\hline 1985 & 1477 & 1992 & 920 & 1968 & 906 & 2001 & 1098 & 1961 & 1202 \\
\hline 1989 & 1513 & 2003 & 920 & 1964 & 943 & 1977 & 1130 & 1981 & 1229 \\
\hline 1975 & 1519 & 1994 & 947 & 1977 & 955 & 1961 & 1131 & 1997 & 1241 \\
\hline 1972 & 1523 & 1962 & 948 & 1966 & 968 & 1992 & 1141 & 1990 & 1292 \\
\hline 1982 & 1562 & 1975 & 964 & 1983 & 978 & 1973 & 1153 & 1967 & 1298 \\
\hline 1984 & 1562 & 1996 & 995 & 1995 & 980 & 1972 & 1214 & 2000 & 1327 \\
\hline 1961 & 1599 & 1965 & 996 & 1973 & 985 & 1991 & 1223 & 1991 & 1409 \\
\hline 1965 & 1626 & 1968 & 1005 & 1961 & 989 & 2000 & 1245 & 1965 \\
\hline 1992 & 1663 & 1967 & 1038 & 1998 & 1015 & 1989 & 1257 & 1966 & 1462 \\
\hline 1994 & 1665 & 1991 & 1044 & 1979 & 1016 & 1981 & 1270 & 1973 & 1467 \\
\hline 1998 & 1741 & 1986 & 1056 & 2000 & 1016 & 1998 & 1278 & 1986 \\
\hline 1990 & 1933 & 1981 & 1106 & 1996 & 1022 & 1985 & 1403 & 1998 & 1537 \\
\hline 1996 & 2007 & 2000 & 1108 & 1992 & 1047 & 1996 & 1452 & 1996 & 1582 \\
\hline 1973 & 2015 & 1985 & 1132 & 1980 & 1048 & 1986 & 1581 & 1992 & 1603 \\
\hline 2002 & 2090 & 1980 & 1242 & 2002 & 1216 & 2002 & 1591 & 2002 & 1733 \\
\hline 1986 & 2218 & 2002 & 1301 & 1986 & 1427 & 1984 & 1715 & 1994 & 1908 \\
\hline
\end{tabular}

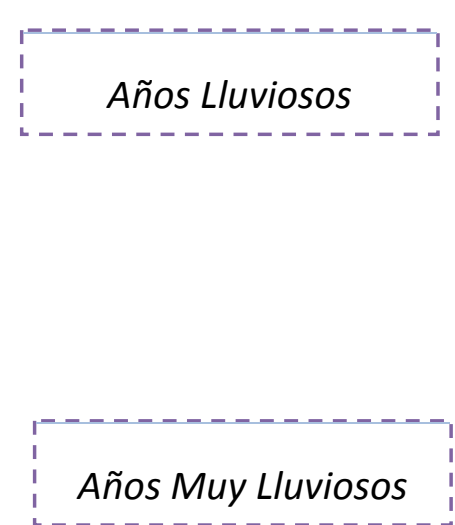

Publicado en formato digital: Lic. Oscar Luis Pyszczek y Prof. María Emilia Pérez.. LAS PRECIPITACIONES Y LOS PERíODOS HÚMEDOS EN LA PROVINCIA DEL CHACO ENTRE LOS AÑOS 1961 Y 2005. Revista Geográfica Digital. IGUNNE. Facultad de Humanidades. UNNE. Año 10. № 19. Enero - Junio 2013. ISSN 1668-5180 Resistencia, Chaco. En: http://hum.unne.edu.ar/revistas/geoweb/default.htm 